\title{
Correlation of p53 Status with Histopathological Grading of Glial Tumors
}

\section{IJCRR}

Section: Healthcare

Sci. Journal Impact

Factor: $6.1(2018)$

ICV: 90.90 (2018)

(c) (i) (8)

Copyright@IJCRR

\section{Tabish Hassan¹, Sunita Vagha², Samarth Shukla³, Amoli Belsare ${ }^{4}$}

\section{ABSTRACT}

Introduction: Glial tumours account for $70 \%$ of all brain tumours with astrocytoma (38.7\%) being the most frequent tumour. The prognosis of high-grade gliomas is very poor and have a limited overall survival rate.

Aim: To establish the role of p53(immunohistochemical) with histopathological grades of various glial tumours such as astrocytoma, oligodendroglioma and ependymoma.

Methods: This will be a Prospective analytical Study. The study will be carried out from 1st August 2018 to 31 st July 2020 in the Histopathology division and Immunohistochemistry section in the Department of Pathology, Jawaharlal Nehru Medical College (J.N.M.C) in co-ordination with Department of Neuro-Surgery. A total of 50 resected specimens of radiologically suspected cases of Glial tumours operated in the Department of Neurosurgery will be considered for the study. The resected specimens will be grossed, processed and evaluated for histopathology and p53 expression.

Results: A direct correlation between the histopathological grade and the p53 expression will be observed.

Conclusion: High-grade gliomas exhibit high p53 expression. Thus, p53 as an adjunct to histological grade can provide a supportive clue to the clinicians to predict the biological behaviour of gliomas.

Key Words: Glial tumour, p53, Immunophenotyping

\section{INTRODUCTION}

Glioma incidence in India ranges from 6 to 12 per 100,000 population. Glial tumours account for $70 \%$ of all brain tumours with astrocytoma (38.7\%) being the most common primary tumour. ${ }^{1-2}$ In the adult population, most of the tumours are high-grade gliomas. In the pediatric age group, the most common tumour is also astrocytoma but was low grade such as pilocytic astrocytoma and subependymal giant cell astrocytoma. Histological grading of gliomas shows in-depth invasion within the brain parenchyma. However, it's currently absolutely clear that data on the molecular understanding usually results in a lot of study for classification of (glial) neoplasms. Pilocytic astrocytoma (PA) is a biologically and histologically distinct type of astrocytoma found in young kids and is the most frequent tumour of the brain in children. Most PAs arise within the neural structure and chiasma. Some arise within the cerebral hemispheres and alternative locations. Diffuse astrocytomas are more common in adolescents. The origin of this tumour can be from anywhere but they are seen in the cerebral hemispheres frequently, particularly frontal lobes. They are difficult to see and it is difficult to ascertain where they begin or end. Anaplastic astrocytoma is clinically and pathologically an intermediate entity between Grade-II Diffuse astrocytoma and glioblastoma. The grade II Diffuse astrocytoma however it has a lot more cellularity and has a lot more atypia and a larger mitotic rate.

Glioblastoma multiforme happens in people between the ages of 40-60 years. Most common sites are the frontal and temporal lobes, however, it has been shown that this tumour can occur at any age and arise from anywhere in the brain. Some glioblastomas originate through the malignant changes occurring within the inferior astrocytomas (secondary glioblastoma). Primary glioblastomas tend to be very dangerous especially if it happens in the elderly. The survival rate of

\section{Corresponding Author:}

Tabish Hassan, 2nd Year, Department of Pathology, Jawaharlal Nehru Medical College, Datta Meghe Institute of Medical Sciences (Deemed to be University), Sawangi (Meghe), Wardha, Maharashtra, India; Contact: 9934957319; Email: tabish00777@gmail.com

ISSN: 2231-2196 (Print)

Received: 17.09 .2020
ISSN: 0975-5241 (Online)

Revised: 22.10 .2020
Accepted: 09.11 .2020
Published: 30.11 .2020 
this tumour is poor and therapy such as surgery, radiation and medication are often in vain. On examination, glioblastoma may be a poorly outlined intra-axial mass with varicolored (multiform) attributed to necrosis and haemorrhage. If the tumour is nearby the centre it can travel from corpus callosum to one hemisphere to the next. Histologically, the tumour presents with nuclear atypia, hypercellularity, and high-grade mitotic activity. It also shows increased microvascular proliferation and serpentine pattern of necrosis.

The prognosis of high-grade gliomas is very poor and have a low survival rate. Gliomas are very difficult when it comes to treatment. Researchers have tried hard to understand the cell physiology and genetic alteration in the cell so we know more about the tumours and make proper effective treatment. Many oncogenes and their protein products show involvement. One of them happens to be p53. The p53 is found at the short arm of chromosome 17 . The p53 gene is of two types wild type and mutant type. .,4 $^{37}$

With the help of immunohistochemistry, we can check for abnormal p53 in the tumour. Staining is dependent on the half-life of mutant p53. If p53 is found in the cell whether it be wild or mutant, the will show positivity.

\section{OBJECTIVE}

To study the histological grade of glial tumours and the immunoexpression of $\mathrm{p} 53$.

\section{MATERIALS AND METHODS}

This is prospective analytical study initiated on 1st August 2018 and completed on 31st July 2020 in the Histopathology division and Immunohistochemistry section in the Department of Pathology, Jawaharlal Nehru Medical College (J.N.M.C) in co-ordination with Department of Neuro-Surgery.

A total of 50 resected specimens of radiologically suspected cases of Glial tumours operated in the Department of Neurosurgery, A.V.B.R.H, Sawangi (Meghe), Wardha will be considered for the study. The resected specimens will be grossed, processed and evaluated for histopathology and p53 expression.

\section{Inclusion Criteria}

- Patients diagnosed with glial tumours on histopathology.

- Patients of both genders.

- Patients of all ages.

\section{Exclusion Criteria}

- Cases of glial tumours with other associated tumours diagnosed on histopathology.

- Patients on radiotherapy and chemotherapy.

- Cases of recurrence.

\section{Statistical Analysis}

The analysis would be carried out by using 'Chi-square test'. p53 Grading by Immunohistochemistry: The p53 grading for all the cases will be done by a single senior pathologist who will be blind to the result of histological grade of the malignancy to avoid any kind of bias. ${ }^{5}$

\section{EXPECTED RESULTS}

The data will be put in master-chart and grouped and tabulated according to various criteria. The relationship with the p53 expression and histopathological grade of the gliomas will be assessed and studied. The sensitivity and specificity will be calculated and analysed.

\section{DISCUSSION}

The nuclear positivity will show the expression of p53 in the tumour.

P53 scoring system- p53 is scored from 0 to 3

No stain in the nucleus

$10 \%$ stain of the nucleus

$10-30 \%$ stain of the nucleus

$>30 \%$ stain of the nucleus

The localization of the p53 gene to the short arm of chromosome 17 is of interest because there is a common pattern of loss of one the alleles in the astrocytoma of the brain. In recent past that has studied both $\mathrm{p} 53$ gene mutations and $\mathrm{LOH}$ on chromosome $17 \mathrm{p}, 65 \%$ of tumours with p53 mutation suffer a loss of chromosome $17 \mathrm{p}$. Various mutations have been found in both low and high-grade gliomas. Approximately $95 \%$ of mutations happen in those codons which are more preserved. This may cause a loss of transcription ability due to the mutated p53.

Cancers with p53 mutation show more aggressive tumour behaviour such as widespread metastasis and very less cellular differentiation. A better knowledge of the factors and mechanisms will show aggressive behaviour of some glial tumours. The discussion will be programmed to compare the discoveries of the current study with the observations available in the different studies. p53 show a variety of malignancies including brain tumours. Mutations of p53 are common phenomena of these tumours and through various studies, it has been found out that they can be encountered frequently. ${ }^{6-9}$

Missense mutations are seen in the P53 gene. High expression of p53 can be used as a surrogate marker for p53 deactivation. ${ }^{10}$ However, too high expression of p53 can harm or eventually cause a response that is physiological in response 
to DNA damage. ${ }^{11}$ The observations performed over these objectives of p53 expression will be compared with available observations in the literature for the conclusion of correlation of p53 expression with histological grade of glial tumours. Several articles related to different aspects of cancers were reviewed. ${ }^{12-14}$ Deshpande et al reported on methylation pattern of retrotransposons as a biomarker for human cancer. ${ }^{12}$ Mohite et al conducted an immunohistochemical evaluation of the expression pattern of p53, p63, and p73 in epithelial dysplasia. ${ }^{15}$ Shweta et al reflected on the immuno-expression profile of cyclin D1, Ki67 and P53 in the evaluation of endometrial hyperplasia. ${ }^{16}$

\section{CONCLUSION}

p53 as an adjunct to histological grade can provide a supportive clue to the clinicians to predict the biological behaviour of gliomas.

\section{ACKNOWLEDGMENT}

Authors acknowledge the immense help received from the scholars whose articles are cited and included in references to this manuscript. The authors are also grateful to authors / editors / publishers of all those articles, journals, and books from which the literature for this article has been reviewed and discussed.

\section{Conflict of Interest: Nil}

Source of Funding: Nil

\section{REFERENCES}

1. Nair MK, Varghese C, Swaminathan R. Cancer: Current Scenario, Intervention Strategies and Projections for. NCMH Background Papers Burden of Disease in India. (2015).
2. Yeole BB. Trends in brain cancer incidence in India. Asian Pac J Cancer Prev 2008;9:267-70.

3. Dowell SP, Hall PA. The p53 tumour suppressor gene and tumour prognosis: is there a relationship? J Pathol 1995;177(3):221-4.

4. Dirks PB, Rutka JT, Dirks PB. Current concepts in neuro-oncology: the cell cycle-a review. Neurosurgery 1997;40(5):1000-15.

5. Shanmugavadivu AC, Deepika T, Hemamalini R, Madhusudhanan G, Indhu K, Babu NR. p53 Expression in Gliomas. Int J Appl Sci Biotech 2015;3(3):479-82.

6. Levidou G, El-Habr E, Saetta A, et al. p53 immunoexpression as a prognostic marker for human astrocytoma: a meta-analysis and review of the literature. J Neuro Oncol 2010;100:363 -71.

7. Sarkar C, Karak A, Nath N, et al. Apoptosis and proliferation: correlation with p53 in astrocytic tumors. J Neuro Oncol 2005;73:93 -100.

8. Sarkar C, Mukhopadhyay S, Sharma M. p53 in brain tumors: basic science illuminates clinical oncology. Indian J Human Genet 2002;8:52.

9. Litofsky N, Recht L. The impact of tumor suppressor gene on glioma biology. Neurosurg Focus 1997;3:101.

10. Cordon-Cardo C, Dalbagni G, Saez GT, et al. p53 mutations in human bladder cancer: genotypic versus phenotypic patterns. Int J Cancer 1994; 56:347-353.

11. Li M, Brooks CL, Kon N, Gu W. A dynamic role of HAUSP in the p53-Mdm2 pathway. Mol cell 2004;13(6):879-86.

12. Deshpande P, Gupta V, Bhake A. Methylation pattern of retrotransposons: Biomarker for human cancer. J Datta Meghe Inst Med Sci Uni 2018;13(1):66.

13. Khatib MN, Gaidhane A, Gaidhane S, Quazi ZS. Ghrelin as a promising therapeutic option for cancer cachexia. Cell Physiol Biochem 2018;48(5):2172-88.

14. Khatib MN, Shankar AH, Kirubakaran R, Gaidhane A, Gaidhane S, Simkhada P, et al. Ghrelin for the management of cachexia associated with cancer. Cochrane Database of Syste Rev 2018(2).

15. Mohite DP, Hande AH, Gupta R, Chaudhary MS, Mohite PM, Patil S, et al. Immunohistochemical evaluation of expression pattern of p53, p63, and p73 in epithelial dysplasia. J Datta Meghe Inst Med Sci Uni 2018;13(3):122.

16. Shweta P, Bhake A, Sunita V, Singh K. The Immunoexpression Profile of Cyclin D1, Ki67 and P53 in Evaluation of Endometrial Hyperplasia State and Endometrial Carcinoma. Int J Pharma Res 2019; 11 (1):1203. 